\section{ITAS NEWS}

\section{Helmholtz-Allianz präsentiert Ergebnisse}

Die Energiewende ist mehr als der Ersatz von alter durch neue Technologie. Sie ist eine gesellschaftliche Transformation, die viele Lebensbereiche betrifft. Mit dieser Ausgangsvermutung hat die Helmholtz-Allianz ENERGY-TRANS über einen Zeitraum von fünf Jahren die vielfältigen Schnittstellen zwischen technischen, wirtschaftlichen und gesellschaftlichen Faktoren untersucht, die den Umbauprozess hin zu neuen Infrastrukturen bestimmen. Vier Helmholtz-Zentren, vier Universitäten und das Zentrum für Europäische Wirtschaftsforschung waren an der vom ITAS koordinierten Forschungsarbeit beteiligt.

Die Wissenschaftler konnten u. a. belegen, dass Anwohner Entscheidungen über Infrastrukturprojekte eher mittragen, wenn intensive partizipative Verfahren vorgeschaltet werden. In der Regel können Projekte wie neue Stromtrassen oder Wind- und Solarparks von der speziellen Expertise der Bürger vor Ort sogar profitieren. Wichtig ist dabei, Beteiligungsprozesse konstruktiv in Planungsverfahren zu integrieren.

Als aktive Konsumenten sind die Bürger gefordert, selbst zum Gelingen der Energiewende beizutragen. In einem Feldversuch haben die Forscher Haushalte mit Smart Metern ausgestattet und diese mit einem von der Universität Stuttgart entwickelten Smart-Meter-Webportal kombiniert. Es zeigte sich, dass ein System, das Konsumenten vielseitig dabei unterstützt, Strom im Alltag effizient zu nutzen, zu einer deutlichen Energieeinsparung in den Haushalten führen kann.

Ihre Arbeitsergebnisse präsentierten die Wissenschaftler der Helmholtz-Allianz ENERGY-TRANS am 14. und 15. März 2016 bei der Abschlusskonferenz ,Zukünftige Infrastrukturen der Energieversorgung. Auf dem Weg zur Nachhaltigkeit und Sozialverträglichkeit" in Berlin. Ergänzend zu den wissenschaftlichen Vorträgen standen die Themen ,Aktive Bürger und Konsumenten als Chance für die Energiewende" und „Steuerung der Energiewende - wie weiter?“ im
Mittelpunkt zweier Diskussionsrunden mit Vertretern aus Politik, Wirtschaft und Gesellschaft. Info: http://www.energy-trans.de/

\section{$\ll 》$}

\section{Neue stellvertretende Institutsleiterin}

Constanze Scherz wurde vom Institutsleiter des ITAS, Armin Grunwald, ab Januar 2016 zur stellvertretenden Institutsleiterin ernannt. Constanze Scherz ist seit 2005 wissenschaftliche Mitarbeiterin im ITAS. Zuvor arbeitete sie mehrere Jahre im vom Institut betriebenen Büro für Technikfolgen-Abschätzung beim Deutschen Bundestag (TAB) in Berlin. Die Sozialwissenschaftlerin studierte an der Humboldt-Universität zu Berlin und leitet im ITAS seit mehreren Jahren die Redaktion der TATuP. Die Schwerpunkte ihrer wissenschaftlichen Arbeit liegen in den Themenfeldern parlamentarische Politikberatung, Wissen für Entscheidungsprozesse sowie der regelmäßigen Durchführung einer internationalen Summer School für Doktoranden aus den Sozialwissenschaften.

Der Wechsel in der Leitung des Instituts stand an, da der bisherige zweite Institutsleiter Michael Decker zum 1. Oktober 2015 die Leitung des Bereichs II „Informatik, Wirtschaft und Gesellschaft" des Karlsruher Instituts für Technologie (KIT) übernommen hat.

\section{《»}

\section{Kooperationen}

\section{Zusammenarbeit mit russischen Universitäten}

Das ITAS hat neue Kooperationen mit zwei langjährigen russischen Partnern gestartet. Entsprechende Vereinbarungen wurden mit der Tomsker Staatlichen Universität (TSU) in Sibirien und der Polytechnischen Universität Perm (PSTU) im Ural getroffen. Direkte Partner in Tomsk sind der Lehrstuhl von Irina Chernikova am Institut für Philosophie, wo im September 2015 ein internationaler Workshop zum Thema „Social Assessment of Technology and Technoscience" durchgeführt wurde, und das Bildungszentrum „Policy Analysis and Studies of Technology“ (PAST). 
In Perm ist der unmittelbare Partner der Lehrstuhl für Philosophie und Recht mit dem Forschungslabor RRI-Lab unter Leitung von Elena Seredkina. Die Zusammenarbeit wird einen Schwerpunkt in der universitären Lehre zur TA haben, vor allem im Hinblick auf die Ingenieursausbildung. Ein erster Schritt ist die Erarbeitung mehrsprachiger Lehrmaterialien $\mathrm{zu}$ diesem Zweck. Gemeinsame Workshops und die ITAS-Beteiligung an einer Konferenz in Perm Ende 2016 werden folgen. Die Kooperation mit beiden Partnern soll auch zum Entstehen eines russischen TA-Netzwerks beitragen.

\section{Partnerschaft mit dem Orient-Institut Istanbul}

„Mensch, Medizin und Gesellschaft" stehen im Mittelpunkt der Kooperation des ITAS mit dem Orient-Institut Istanbul, einem turkologischen und regional-wissenschaftlichen Forschungsinstitut im Verbund der Max Weber Stiftung. Das Orient-Institut Istanbul baut gerade einen von Melike Şahinol geleiteten Forschungsschwerpunkt zu „Mensch, Medizin und Gesellschaft“ auf. Im Mittelpunkt seiner Forschung steht der spezifische gesellschaftliche Umgang mit Praktiken und Techniken der menschlichen Optimierung durch medizinische Innovationen. Dazu gehören insbesondere aktuelle Themen, wie zum Beispiel Human Enhancement, Genetic Engineering, moderne Reproduktionstechnologien und Gesundheitsapps, die unterschiedliche Dimensionen individuellen und sozialen Lebens verändern.

Beim Aufbau des Forschungsbereichs arbeitet das Orient-Institut Istanbul mit dem ITAS als zentralem Kooperationspartner zusammen. Die Kooperation schließt gemeinsame Workshops, Konferenzteilnahmen, wissenschaftlichen Forschungsprojekte und Publikationsvorhaben ein. Zum offiziellen Start der Partnerschaft fand am 7. Dezember 2015 ein Festakt im ITAS statt. Bei der Veranstaltung sprach Joachim Boldt, der stellvertretende Direktor des Instituts für Ethik und Geschichte der Medizin an der Universität Freiburg, über die „Ethische Debatte um die Verbesserung des Menschen mit medizinischen Mitteln“.

\section{Neue Projekte}

\section{Technologie für flüssigen Solartreibstoff}

Mit der Nachhaltigkeitsanalyse eines neuartigen flüssigen Algenkraftstoffs beschäftigt sich das ITAS in dem neuen Projekt „Photofuel“. Die Wissenschaftler bewerten dabei sämtliche Produktionsschritte, von der Herstellung und Kultivierung eines Algenstrangs bis hin zur Verbrennung des fertigen Kraftstoffs im Motor auf ökologischer, ökonomischer sowie sozialwissenschaftlicher Ebene. Das Ziel von Photofuel besteht darin, eine neuartige Technologie zur Produktion von flüssigem Solartreibstoff zu entwickeln.

Das vom EU-Programm Horizon 2020 geförderte Projekt wird vier Jahre lang von einem europäischen Konsortium mit Partnern aus Industrie und Wissenschaft durchgeführt. Die Arbeit des interdisziplinär zusammengesetzten Teams aus Ingenieuren, Natur- und Sozialwissenschaftlern ist in sieben Arbeitspakete unterteilt.

Unter der Leitung von ITAS werden in Kooperation mit IFP Energies nouvelles (IFPEN), VW und Volvo ökologische und ökonomische Auswirkungen der Photofuel-Technologie abgeschätzt sowie gesellschaftliche Risiken identifiziert. Im Mittelpunkt des Arbeitspakets stehen prospektive Lebenszyklusanalysen (LCA), um Photofuel mit bestehenden Technologien der Kraftstoffproduktion aus fossilen und biogenen Energieträgern zu vergleichen. Den zweiten Schwerpunkt bilden sozialwissenschaftliche Untersuchungen der Wahrnehmung von Chancen und Herausforderungen der Photofuel-Technologie mittels Stakeholder-Workshops.

\section{Kontakt}

Dr. Christine Rösch

E-Mail: christine.roesch@kit.edu

http://www.itas.kit.edu/num_lp_roes15_pfuel.php

\section{Begleitforschung zu Bioökonomie}

Das Land Baden-Württemberg hat mit seiner Forschungsstrategie „Bioökonomie im System aufstellen“ im Jahr 2013 die Weichen für ein umfangreiches Forschungsprogramm zur Nutzung biobasierter Rohstoffe in den Bereichen Lignozellulose, Biogas und Mikroalgen gestellt. Das Land 
fördert über 40 Projekte überwiegend naturwissenschaftlich-technischen Charakters, aber auch in den Bereichen Modellierung, Potenzialanalyse und Folgenabschätzung. Nun wurde das ITAS damit beauftragt, Ziele und Visionen der beteiligten Forscherteams zu erheben, Veränderungen in den bereits existierenden Wertschöpfungsketten sowie mögliche Folgen für Umwelt und Wirtschaft zu identifizieren, die vorhandenen Akteursbeziehungen zu erfassen, rechtliche Rahmenbedingungen und mögliche Hemmnisse aufzuzeigen sowie alternative Umsetzungspfade zu diskutieren.

Die Ergebnisse sollen einen Beitrag zur Gesamtanalyse des Transformationsprozesses und seiner erwartbaren Implikationen leisten und die Systemsicht im Forschungsprogramm schärfen. Ziel des Projekts ist es außerdem, Orientierung für die weitere Ausgestaltung einer Bioökonomie in Baden-Württemberg zu geben, die sich am Prinzip der Nachhaltigkeit orientiert.

\section{Kontakt}

PD Dr. Rolf Meyer

E-Mail: rolf.meyer@kit.edu

http://www.itas.kit.edu/num_lp_meye15_

biooekonbw.php

\section{STOA-Projekt zu Assistiven Technologien}

Für das EU-Parlament analysiert das ITAS Trends Assistiver Technologien und ihre Auswirkungen auf Menschen mit Behinderungen, insbesondere bezogen auf ihre Lern- und Arbeitsumgebung und ihre gesellschaftliche Integration insgesamt. Dabei werden aktuelle technische Trends und künftige Entwicklungen, sowie regulatorische Rahmenbedingungen für drei verschiedene Formen von Behinderungen (Gehörlosigkeit und Schwerhörigkeit; Blindheit und Sehbehinderung; Autismus) untersucht. Über eine OnlineBefragung und Experteninterviews wollen die Wissenschaftler die Wahrnehmung technischer Unterstützung und die entsprechenden Erwartungen von Menschen mit Behinderungen in den Mitgliedstaaten der EU erheben.

Die so gewonnenen empirischen Ergebnisse werden in einem „Horizon Scanning“ unter Einbindung externer Experten in vier Zukunftsszenarien überführt und filmisch umgesetzt. Das Projekt unterstützt so das Europäische Parlament bei der Förderung Assistiver Technologien für Menschen mit Behinderung und der Gestaltung künftiger rechtlicher Regulierungen in diesem Feld.

\section{Kontakt}

Dr. Linda Nierling

E-Mail: linda.nierling@kit.edu

http://www.itas.kit.edu/wuw_lp_nier16_asstech.php

\section{Machbarkeit, Chancen und Hemmnisse urbaner Luftseilbahnen}

Seit einigen Jahren diskutieren Verkehrsexperten und Stadtplaner immer wieder über den Einsatz von Luftseilbahnen im Stadtgebiet als Ergänzung zum öffentlichen Nahverkehr. In Deutschland sind bisher jedoch die meisten dieser Vorhaben gescheitert. Trotz technisch voll ausgereifter Systeme stoßen die neuen Projekte teilweise auf erhebliche Widerstände in der Bevölkerung und unter professionellen Akteuren. In dem neuen Projekt sollen die vorgebrachten Argumente und Positionen für und wider urbane Luftseilbahnsysteme transparent aufbereitet werden.

Dabei werden sowohl gescheiterte als auch realisierte Vorhaben in Deutschland untersucht und in Experteninterviews die Rahmenbedingungen identifiziert, die zum Gelingen oder Scheitern beigetragen haben. Die aus diesen Interviews gewonnenen Erkenntnisse dienen dazu, drei Untersuchungsräume in Baden-Württemberg zu identifizieren, die sich potenziell für Seilbahnlösungen anbieten würden. In diesen drei Regionen werden die Erwartungen und Bedenken in Workshops mit Wissenschaftlern und professionellen Akteuren identifiziert und diskutiert sowie Fokusgruppen mit Bürgern zum Thema durchgeführt. Zusätzlich werden mithilfe eines mikroskopischen Verkehrsmodells die anzunehmenden Wirkungen einer Luftseilbahn in einem der potenziellen Untersuchungsräume quantitativ analysiert, um eine gesamtökonomische und ökologische Bewertung vorzunehmen.

\section{Kontakt}

Maike Puhe

maike.puhe@kit.edu

http://www.itas.kit.edu/iut_lp_puhe16_hohibawu.php

《» 
Personalia

Prof. Dr. Armin Grunwald wurde in die Arbeitsgruppe „Mit Sicherheit mehr Sicherheit? Perspektiven für die Sicherheitspolitik der Welt in 2035“ berufen, die von der Nationalen Akademie der Wissenschaften Leopoldina finanziert wird. Diese Arbeitsgruppe wird vom Forum für Friedensund Konfliktforschung der Hamburger Akademie getragen und umfasst Mitglieder aus mehreren wissenschaftlichen Akademien. Die Aufgabe der AG ist es, jenseits der tagespolitischen Herausforderungen der Sicherheitspolitik über langfristige Entwicklungen nachzudenken und hierfür Orientierung für vorausschauendes politisches Handeln zu erarbeiten. Ihr Bericht soll im Jahr 2017 der Öffentlichkeit vorgestellt werden.

Daniel Ketzer hat mit seinem Vortrag zu Agrophotovoltaik und der Vorstellung des Projekts APV-RESOLA den „Best Presentation Award" im Forum Primärrohstoffe beim Nachwuchsforscherkongress ,24 h für Ressourceneffizienz" in Pforzheim erhalten. Besonders die klare Herleitung zum Thema Flächennutzungskonflikte zwischen Landwirtschaftssektor und Energiewirtschaft sowie die Vorstellung des ganzheitlichen Forschungsansatzes wurden bei der Auszeichnung hervorgehoben.

Maria João Maia ist nach zwei Jahren als Gastwissenschaftlerin mit einem Stipendium des KIT seit März 2016 wissenschaftliche Mitarbeiterin am ITAS. Mit ihrem gesundheitswissenschaftlichen Hintergrund forscht sie an TAThemen aus diesem Bereich. Aktuell arbeitet sie im Projekt „Assistive Technologien für die Inklusion von Menschen mit Behinderungen in Gesellschaft, Bildung und Arbeitsmarkt".

Helena Trenks unterstützt seit Januar 2016 das ITAS Forschungsprojekt „Quartier Zukunft - Labor Stadt“ sowie das „Reallabor R131“. Sie hat zuletzt den Diplomstudiengang der Landschaftsplanung und Landschaftsarchitektur an der Universität für Bodenkultur Wien abgeschlossen. Sie wird sich vor allem mit Fragen der nachhaltigen Freiraumentwicklung im Quartier sowie mit Möglichkeiten und Formen der Bürgerbeteiligung im Zuge des Reallabors befassen.
Neue Veröffentlichungen

\section{Alter und Technik}

Das Phänomen einer immer älter werdenden Gesellschaft ist zu einer großen sozialen Herausforderung geworden. Dabei haben technische Entwicklungen in den letzten Jahren dazu beigetragen, das Leben älterer Menschen in vielen Bereichen zu erleichtern. In aktuellen Debatten um Assistive Technologien und ältere Menschen als Nutzer von Technik wird der ,menschliche Faktor" allerdings häufig noch immer ignoriert.

Der Sammelband rückt die Perspektive der Menschen, die von der Entwicklung und dem Gebrauch von Technologien betroffen sind, in den Mittelpunkt. Dieser Perspektivenwechsel den Menschen und nicht die Technik an erste Stelle zu setzen - sensibilisiert für die ambivalenten Effekte der Techniknutzung und soll dabei helfen, Technologien auf die tatsächlichen Bedürfnisse auszurichten und so zu einer Verbesserung der Lebensqualität älterer Menschen beizutragen.

Bibliografische Angaben: Domínguez-Rué, E.; Nierling, L.: Ageing and Technology. Perspectives from the Social Sciences. Bielefeld: transcript 2016, ISBN 978-3-8376-2957-6

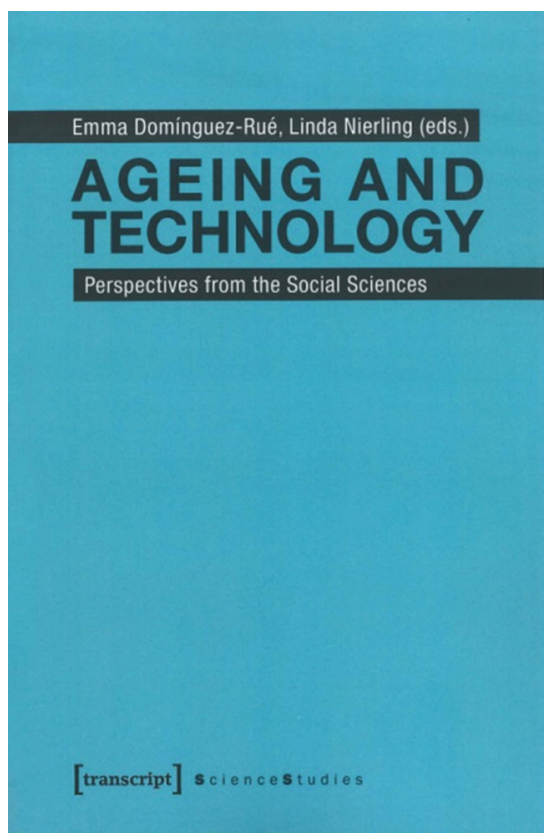




\section{PACITA-Konferenzband}

Die zweite europäische TA-Konferenz markierte den Abschluss des EU-Projekts PACITA (Parliaments and Civil Society in Technology Assessment), das sich mit Methoden, Praktiken und Institutionen für wissensbasierte Politikberatung rund um Themen wie Wissenschaft, Technologie oder Innovation auseinandersetzte.

Der Fokus des jetzt erschienenen Bands liegt, analog zur Konferenz, auf der Rolle von TA und ihren aktuellen und zukünftigen Herausforderungen. Gerade die so genannten „Grand Challenges“, wie der demografische Wandel, nachhaltige Energie oder Mobilität, Klimawandel und Ressourceneffizienz oder Freiheit und Sicherheit von Bürgern sind drängende Themen, zu denen die TA einen wichtigen Beitrag leisten kann. Als interdisziplinäres, politikberatendes und die Öffentlichkeit beteiligendes Konzept kann sie helfen, diese Herausforderungen besser zu verstehen und Optionen anbieten, um sie zu bewältigen.

Die online verfügbaren Proceedings versammeln den Großteil der präsentierten Vorträge und beinhalten Zusammenfassungen spezieller Formate wie Round Table- oder PanelDiskussionen während der Konferenz.

Bibliografische Angaben: Scherz, C.; Michalek, T.; Hennen, L.; Hebáková, L.; Hahn, J.; Seitz, S.B. (Hg.): The Next Horizon of Technology Assessment. Proceedings from the PACITA 2015 Conference in Berlin. Prague: Technology Centre ASCR 2015, ISBN 978-80-7333-121-4; Link zum PDF-Download unter http://www.itas.kit.edu/2016_018.php

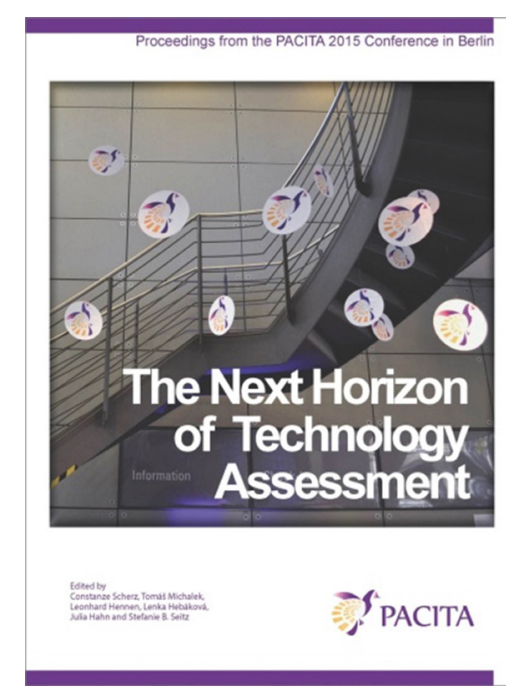

\section{Informationen zum ITAS}

Das Institut für Technikfolgenabschätzung und Systemanalyse (ITAS) im Karlsruher Institut für Technologie erarbeitet und vermittelt Wissen über die Folgen menschlichen Handelns und ihre Bewertung in Bezug auf die Entwicklung und den Einsatz von neuen Technologien. Alternative Handlungs- und Gestaltungsoptionen werden entworfen und bewertet. ITAS unterstützt dadurch Politik, Wissenschaft, Wirtschaft und die Öffentlichkeit, Zukunftsentscheidungen auf der Basis des besten verfügbaren Wissens und rationaler Bewertungen zu treffen. $\mathrm{Zu}$ diesem Zweck wendet ITAS Methoden der Technikfolgenabschätzung und Systemanalyse an und entwickelt diese weiter. Untersuchungsgegenstände sind in der Regel übergreifende systemische Zusammenhänge von gesellschaftlichen Wandlungsprozessen und Entwicklungen in Wissenschaft, Technik und Umwelt. Das Institut erarbeitet sein Wissen vor dem Hintergrund gesellschaftlicher Probleme und Diskurse sowie anstehender Entscheidungen über Technik. Relevante gesellschaftliche Akteure werden in den Forschungs- und Vermittlungsprozess einbezogen. Außerdem greift das ITAS die Problematik der Bewertung von Technik und Technikfolgen mit wissenschaftlichen Mitteln auf. Die Forschungsarbeiten des Instituts haben grundsätzlich einen prospektiven Anteil. Es geht - im Sinne der Vorsorgeforschung - um Vorausschau der Folgen menschlichen Handelns, sowohl als Vorausschau soziotechnischer Entwicklungen (Foresight) als auch als Abschätzung künftiger Folgen heutiger Entscheidungen. Als Richtschnur gilt, dass die Forschungsergebnisse in unterschiedlichen, alternativen Handlungs- und Gestaltungsoptionen gebündelt und in Bezug auf ihre Folgen und Implikationen rational bewertet werden. Das Internetangebot des Instituts finden Sie unter http://www.itas.kit.edu. 